\title{
Tunable single-electron tunneling behavior of ligand-stabilized gold particles on self-assembled monolayers
}

\author{
Bing Wang, Haiqian Wang, Huixiang Li, Changgan Zeng, and J. G. Hou* \\ Structure Research Lab, Center for Physical Sciences, University of Science and Technology of China, Hefei 230026, Anhui, China \\ Xudong Xiao \\ Department of Physics, The Hong Kong University of Science and Technology Clear Water Bay, Kowloon, Hong Kong, China
}

(Received 14 July 2000; published 22 December 2000)

\begin{abstract}
A series of heptanethiol-stabilized gold particles with narrowly distributed core sizes ranging from $\sim 1.8$ to $\sim 15 \mathrm{~nm}$ were synthesized and deposited on self-assembled alkanethiol monolayers. The current-voltage characteristics were studied systematically by scanning tunneling microscopy and scanning tunneling spectroscopy, and the results correlate well with the gold core sizes. While equidistant staircases originating from the Coulomb blockade $(\mathrm{CB})$ effect are observed for large particles $(\geqslant 4.6 \mathrm{~nm})$, irregular staircases are observed for small particles (nominal size of $1.8 \mathrm{~nm}$ ). The latter indicates that both the $\mathrm{CB}$ effect and discrete quantum energy levels contribute to the current-voltage spectra. Furthermore, single-electron tunneling behavior are shown to be tunable either by varying the gold particle size or by adjusting the tip-particle distance.
\end{abstract}

DOI: 10.1103/PhysRevB.63.035403

PACS number(s): 73.23.Hk, 73.22.-f, 61.46.+w, 68.37.-d

\section{INTRODUCTION}

Single-electron tunneling (SET) has been proposed as the basis of digital nano-electronics, including the key components for logic and memory devices. ${ }^{1}$ Many efforts have been made both to reveal the physics of SET (Refs. 2-18) and to fabricate new types of SET devices. ${ }^{19-24}$ As with many other devices, it is useful to develop tunable SET devices for practical use, for which it is essential to have a thorough understanding of the SET behavior in a double barrier tunnel junction (DBTJ) geometry with both the size of the central electrode (particle) and the environmental parameters connecting the particles to the external circuits being adjustable. Scanning tunneling microscopy/scanning tunneling spectroscopy (STM/STS) is a powerful tool for studying the SET behavior of particles with different sizes at nanometer scales in the DBTJ geometry. Moreover, the environmental parameters such as tip-particle distance can be easily adjusted.

Of the many previous studies of the Coulomb blockade (CB) effect, only a few experiments ${ }^{9,13,15,18,22,24,25}$ used particles with well-characterized sizes. It is still a challenge to correlate the SET behavior with the particle size experimentally, even though it is well-known that both the singleelectron charging energy and the discrete quantum energy levels are size dependent. For example, if a particle size varies from 20 to $1 \mathrm{~nm}$, the charging energy is estimated to increase from several $\mathrm{meV}$ to several hundred meV. Meanwhile, the discreteness of the quantum energy levels is expected to be more and more evident, ${ }^{11,13}$ and even atomiclike electronic behavior becomes observable. ${ }^{15,18}$ For very small particles, a tunneling current-voltage $(I-V)$ curve of a DBTJ may mix the Coulomb blockade effect with the discrete quantum energy levels. While the Coulomb blockade effect gives equidistant current steps in the $I-V$ curves, the internal discrete energy levels may give nonequidistant current steps. To correlate these effects directly with the particle size, we need to use a series of well-fabricated conductive particles with mono- or at least narrow-dispersion. The recent success in the fabrication of noble metal particles and semiconductor particles with narrow size distributions using chemical methods $^{26-32}$ provides us an opportunity. The ligandstabilized conductive particles have obvious advantages not only for the relative ease of preparation, but also for their long-term stability, systematic size control and narrow size distribution. These particles may become very good candidates for SET devices, and have attracted wide interest in the past years. For example, the SET behavior of Au, ${ }^{16-18,22,25}$ $\mathrm{Pt}^{13}$ semiconductor InAs (Ref. 15) and CdSe (Refs. 21,24) particles has been well examined. However, the particle size used in these studies only covers a very narrow range, and no attention was paid to the size tuning behavior.

On the other hand, single-electron tunneling can also be tuned by adjusting the environmental parameters connecting the particles to the external circuits. In the previous STM studies, conductive particles are deposited on the support barriers, e.g., oxidation films or organic films. ${ }^{7-10,13,15}$ Sometimes, organic monolayer coated particles are directly deposited on electrode substrates. ${ }^{16,25}$ The dielectric constant of the particle-electrode junction is either much larger or comparable to that of the tip-particle junction. Hence, the capacitance $C_{1}$ of the particle-electrode junction is typically larger than or nearly equal to the capacitance $C_{2}$ of the tip-particle junction, since the tip-particle distance cannot be adjusted to completely compensate for the contribution from the dielectric constant. According to the orthodox theory, ${ }^{3,4}$ the voltage spacing of Coulomb staircases is inversely proportional to the larger capacitance of $C_{1}$ and $C_{2}$. In the case of $C_{1}>C_{2}$, the Coulomb blockade effect is dominated by $C_{1}$, and the spacing of Coulomb staircases cannot be tuned by varying $C_{2}$. Schönenberger et al. ${ }^{7}$ and Andres et al. ${ }^{9}$ illustrated these points. Though the tip-particle distance was varied through adjusting the set point current in their studies, their calculation and fitting results ${ }^{7,9}$ showed that $C_{1}$ was larger than $C_{2}$. If we construct a DBTJ with $C_{1}<C_{2}$, the SET effect may be 
tunable within a certain range by adjusting the tip-particle distance. In order to achieve this, the dielectric constant of the support barrier must be low and its thickness must be relatively large. The structure used by $\mathrm{Chi}$ et al. ${ }^{17}$ with ligand-coated $\mathrm{Au}_{55}$ deposited on a self-assembled organic layer meets the above conditions, but they used a very shortchain thiol molecule and did not pay attention to the tuning behavior.

In this paper, we present a systematic study of tunable single-electron tunneling using STM/STS. The ligand-coated gold particles were well characterized by high-resolution electron microscopy (HREM) with an average core size of $1.8 \sim 15 \mathrm{~nm}$, depending on the fabrication conditions. They were deposited on alkanethiol self-assembled monolayers (SAM's) on gold substrates (SAM/Au) for SET measurement using STM/STS. For large gold particles, the SET behavior correlates well with their size, and can be fully accounted for by the theoretically calculated capacitances. For gold particles with a small core size (nominal $1.8 \mathrm{~nm}$ ), a series of irregularly spaced current steps are observed, which is interpreted as originating from the discrete energy levels due to quantum size effect. By varying the distance between the STM tip and the particle through adjusting the set point current, the voltage spacing of Coulomb staircases can also be tuned within a certain range. As the tip-particle distance decreases, the fitting value of $C_{2}$ increases and becomes larger than $C_{1}$. Our experiment clearly shows that the SET behavior can be tuned through changing the particle size or adjusting the environmental parameters of the particles.

\section{EXPERIMENT}

In the experiment, the SAM substrate was prepared by first evaporating $160 \mathrm{~nm}$ thick gold on freshly cleaved mica sheets heated at $300^{\circ} \mathrm{C}$ to form $\mathrm{Au}$ (111) substrates. Then, a monolayer of decanethiol $\left(\mathrm{C}_{10}\right)$ was self-assembled on the fresh $\mathrm{Au}$ (111) by immersion into a $2 \mathrm{mM}$ ethanol solution of $\mathrm{C}_{10}$ for $48 \mathrm{~h}$. The molecular confirmation in the monolayer was well studied. The molecules tilt away from the surface normal by $30^{\circ}$ and thus form a layer $\sim 1.4 \mathrm{~nm}$ thick, ${ }^{33}$ and give an average area of $22.2 \AA^{2}$ per molecule. ${ }^{34}$ STM images of SAM's indicated that the monolayers are well ordered and densely packed. Electric measurement ${ }^{35}$ revealed that the monolayer is a good insulator with a relatively small dielectric constant of $\sim 2.7$ and a conductivity of $(6 \pm 2) \times 10^{-15}$ $\Omega^{-1} \mathrm{~cm}^{-1}$ for alkanethiol $\left[\mathrm{CH}_{3}\left(\mathrm{CH}_{2}\right)_{n-1} \mathrm{SH}\right]$ with a chain length $n$ from 16 to 34 . Such a molecular monolayer with an atomically flat surface provides both the supporting layer and the tunneling barrier for the metal particles.

Ligand-stabilized gold particles from $\sim 1.8$ to $\sim 15 \mathrm{~nm}$ were synthesized by the well-established two-phase method. ${ }^{27,28}$ Heptanethiol was used as the stabilizing ligand. The details of the method can be found in Refs. 27 and 28. To achieve different particle sizes, the conditions of synthesis were adjusted by changing the $\mathrm{AuCl}^{-}$:heptanethiol [abbreviated as $\mathrm{Au}: R \mathrm{~S}$, here $\left.R=\mathrm{CH}_{3}\left(\mathrm{CH}_{2}\right)_{6}\right]$ molar ratio from $1: 2$ to $6: 1 .^{27-29}$ During the synthesis of large ligandstabilized gold particles, the two-phase $\left(\mathrm{H}_{2} \mathrm{O}+\right.$ tolurene $)$ mixture solution is irradiated by a low-pressure mercury lamp while being lightly stirred. After reaction, the derived clusters in the organic phase were isolated by precipitation following the addition of methanol. The pure products were redissolved in toluene. One droplet of diluted toluene solution of the nanoparticles was spread on a piece of $\mathrm{C}_{10} \mathrm{SAM}$ or a carbon-coated copper mesh grid, allowing slow evaporation of the solvent. The former sample is used for measurement with STM and the latter with HREM. Although the thiol ligands tilt away from the surface normal even more than $30^{\circ}$ because there is a larger free space for the molecules on a curved gold core than on a planar substrate, it may not deviate too much from $30^{\circ}$. Hence, the thickness of the heptanethiol layer outside the gold core is estimated to be about $1.0 \mathrm{~nm} .{ }^{33}$

The size and distribution of the ligand-coated gold particles was characterized with a JOEL 2100 high-resolution electron microscope (HREM) operated at an accelerating voltage of $200 \mathrm{kV}$. In the HREM images, only the Au cores give a contrast, which allows us to identify the size of the $\mathrm{Au}$ particles. The Au particles spread from the same solution on a SAM/Au substrate are assumed to have the same size distribution.

The SET experiments were performed in an OMICRON low-temperature ultrahigh vacuum scanning tunneling microscope operating at $4.2 \mathrm{~K}$. An asymmetric DBTJ structure is formed when an STM tip is positioned above a ligandstabilized gold particle that was deposited on $\mathrm{C}_{10} \mathrm{SAM} / \mathrm{Au}$. In this geometry, the distance between the particle and the gold electrode is relatively large. Since the thickness of $\mathrm{C}_{10}$ SAM is $\sim 1.4 \mathrm{~nm}$, the thickness of the coated ligand is about $\sim 1.0 \mathrm{~nm},{ }^{33,34}$ and the distance between the Au particle and the conductive gold electrode is about $\sim 2.4 \mathrm{~nm}$. Thus, the capacitance $C_{1}$ of the particle-electrode junction may be smaller than the capacitance $C_{2}$ of the tip-particle junction when the distance between the STM tip and the particle becomes small by adjusting the set point voltage or the set point current. To ensure that our STS data were associated with an individual particle, we only selected for measurement particles that were well separated from others.

\section{RESULTS AND DISCUSSION}

When prepared with dilute solution, the ligand-stabilized gold particles are observed well separated from one another in the HREM images. Figure 1 shows four different samples of gold particles prepared under different conditions: (a) $15.2 \pm 2.1 \mathrm{~nm}(6: 1 \mathrm{Au}: R \mathrm{~S}$ molar ratio), (b) $9.4 \pm 1.5 \mathrm{~nm}(4: 1$ $\mathrm{Au}: R \mathrm{~S}$ molar ratio), (c) $4.6 \pm 1.2 \mathrm{~nm}(1: 1 \mathrm{Au}: R \mathrm{~S}$ molar ratio), and (d) $1.8 \pm 0.6 \mathrm{~nm}(1: 2 \mathrm{Au}: R \mathrm{~S}$ molar ratio) in diameter as averaged over 200 particles. These gold particles have relatively narrow size distributions, and each particle has a compact, crystalline gold core. In the case of a core size of $\sim 15.2 \mathrm{~nm}$, nearly all particles are multiple twinned gold crystallites as seen from the atomically resolved HREM images.

The particles on the SAM/Au/mica substrates were measured with STM/STS. In Fig. 2(a), the DBTJ structure formed by the STM Pt/Ir tip above a ligand-stabilized gold particle is depicted. In Fig. 2(b), we show an image of three 

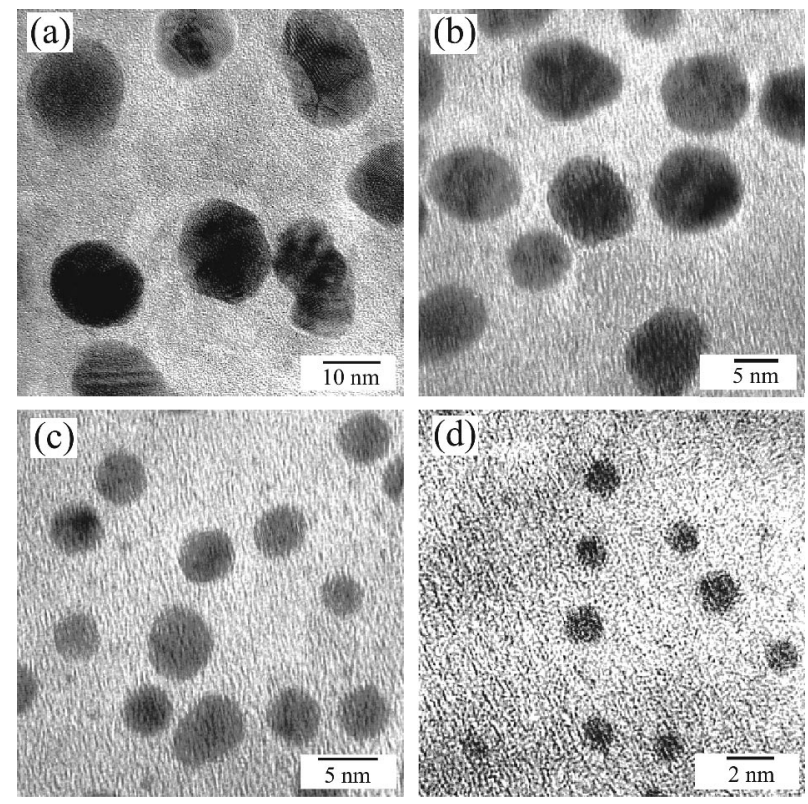

FIG. 1. HREM images of ligand-stabilized gold particles with core sizes of (a) $15 \pm 2.1 \mathrm{~nm}$, (b) $9.4 \pm 1.5 \mathrm{~nm}$, (c) $4.6 \pm 1.2 \mathrm{~nm}$, and (d) $1.8 \pm 0.6 \mathrm{~nm}$ in diameter.

ligand-stabilized $\mathrm{Au}$ particles that are $\sim 10 \mathrm{~nm}$ away from each other. To avoid any complication in data analysis, only such "isolated" particles were selected for the STS. The observed size of the gold particles in the STM images is larger than the actual particle size due to the tip convolution effect and the coated ligand. Thus, we use the HREM result for the average particle sizes. In general, the "isolated" particles observed near defected SAM areas would not move even at a high-imaging current. The Au particles in good SAM areas can be moved by the scanning tip, ${ }^{36}$ possibly due to the very large particle-electrode distance of $\sim 2.4 \mathrm{~nm}$. In such cases, we would directly zoom in on the particle and take $I-V$ curves within a very small scan range of $\sim 1 \mathrm{~nm}$. For a given Au particle, the capacitance $C_{1}$ is fixed, and only $C_{2}$ can be changed by the tip-particle distance through adjusting the set-point current. The capacitance $C_{1}$ can be calculated by the method of image charges if the particles are treated as spheres. For particle diameters of 15.2, 9.4, 4.6, and $1.8 \mathrm{~nm}$, respectively, the calculation gives the capacitance $C_{1}$ as $3.78,2.13,0.91$, and $0.31 \mathrm{aF}$ correspondingly. If $C_{1}$ is the dominant capacitance in the DBTJ, the charging energy would range from $\sim 20$ to $\sim 260 \mathrm{meV}$. Thus, the SET behavior can be tuned within a relatively wide range by varying the particle size.

Several typical $I-V$ curves (thick-solid lines) taken from the particles with different average sizes are shown in Fig. 3, together with their digital differential conductance spectra (dotted lines). It is obvious that the spacing between the adjacent current steps increases with the decrease of particle size. The $I$ - $V$ curves in Figs. 3(a)-3(c) display nearly equidistant current steps, an indication of Coulomb blockade and staircases behavior. These $I-V$ curves can be fitted very well using the orthodox theory. ${ }^{3,4}$ The fitting results are displayed as the thin-solid lines (shifted vertically for clarity) in Figs.
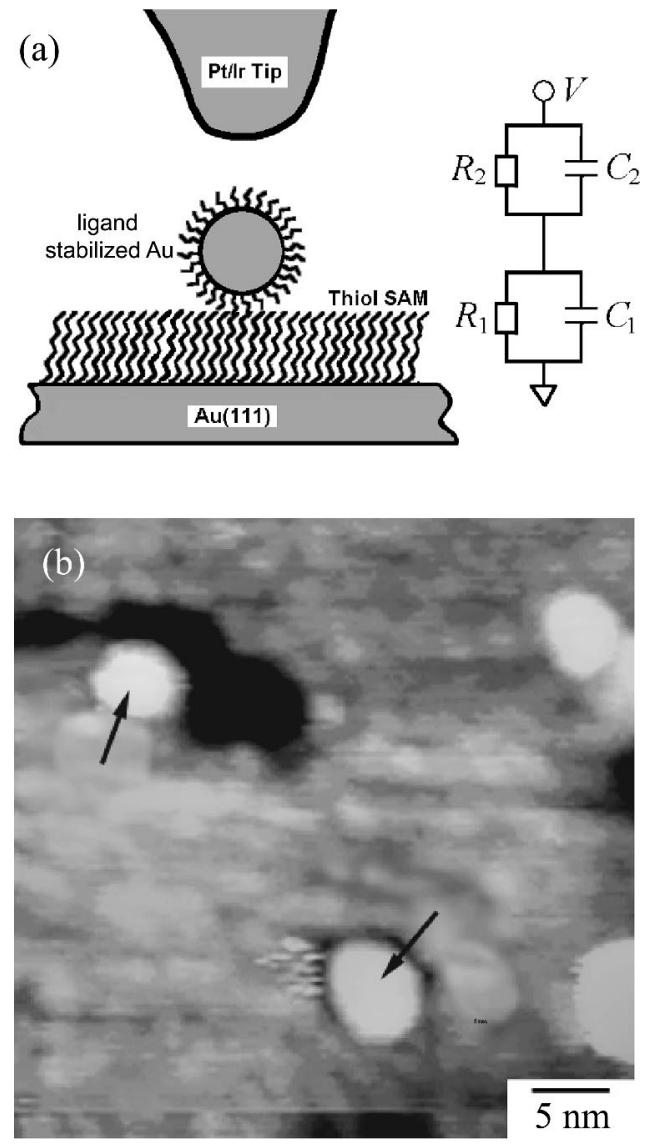

FIG. 2. (a) A schematic of the double-barrier tunneling junctions (DBTJ) formed by an STM tip, a ligand-stabilized gold particle, and a Au substrate. (b) An STM image of gold particles with a nominal core size of $1.8 \mathrm{~nm}$ in diameter. The set point voltage and current are $1.5 \mathrm{~V}$ and $20 \mathrm{pA}$, respectively. The apparent particle size in the image is larger than the real value because of tip convolution and ligand coating.

3(a) $-3(\mathrm{c})$. The fitting parameters $C_{1}\left(C_{2}\right)$ are $4.08(3.95) \mathrm{aF}$ for (a), 2.23 (1.94) aF for (b), and 0.89 (1.16) aF for (c). Here, the fitting values of $C_{1}$ are quite close to the calculated $C_{1}$ values above. It should also be noted that $C_{1}>C_{2}$ for (a) and (b) because of a relatively small set point current ( $2 \mathrm{nA})$, but $C_{1}<C_{2}$ for (c) because of a large set point current (5 nA) which makes the tip closer to the Au particle, and thus, a larger $C_{2}$. We shall discuss the effect of tip-particle distance later. The deviation between the fitting and calculated values of $C_{1}$ is mainly due to the size distribution of particles, which implies that the exact size for the Au particle are unknown under STS measurement.

While the current steps in Figs. 3(a)-3(c) can be attributed to the Coulomb blockade and staircases, the situation is quite different for Fig. 3(d) with a small particle. The $I-V$ curve in Fig. 3(d) has an uneven spacing between the adjacent current steps, with a width of the zero conductance $\sim 1000 \mathrm{mV}$, and a spacing for other current steps of $\sim 210-330 \mathrm{mV}$. A plausible mechanism behind the occurrence of uneven spacing between the adjacent current steps is the internal energy levels originating from the quantum size effect. Similar to semiconductor quantum dots, which dis- 

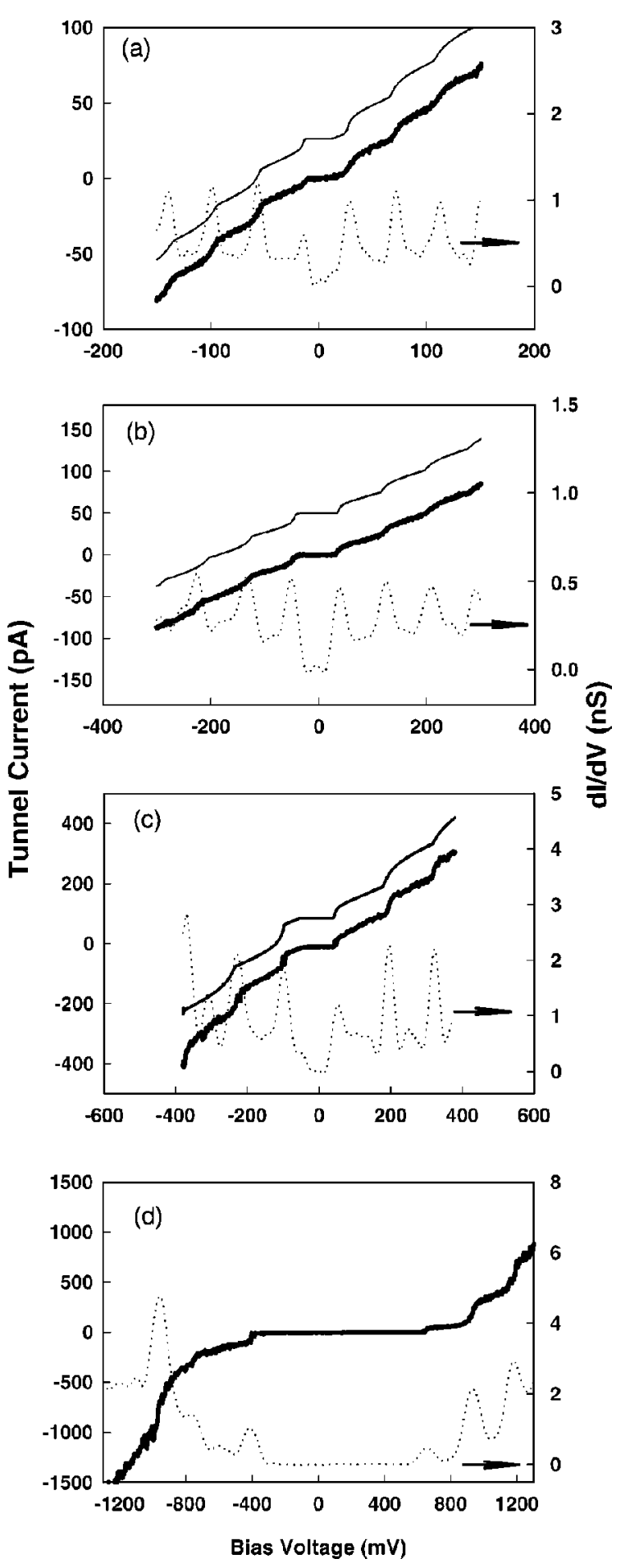

FIG. 3. Four typical $I-V$ curves (thick solid lines) and their digital differential conductance (dotted lines) taken from different samples with nominal particle sizes of (a) $15.2 \mathrm{~nm}$, (b) $9.4 \mathrm{~nm}$, (c) $4.6 \mathrm{~nm}$, and (d) $1.8 \mathrm{~nm}$, respectively. The set point voltages are all $1.5 \mathrm{~V}$, and the set point currents are $2 \mathrm{nA}$ for (a), (b), and (d), but 5 $\mathrm{nA}$ for $(\mathrm{c})$. The parameters for fitted $I-V$ curves (thin solid lines) are $C_{1}\left(C_{2}\right), R_{1}\left(R_{2}\right)$, and $Q_{0}$ : (a) 4.08 (3.95) aF, 69 (1700) $\mathrm{M} \Omega$, and $0.15 e$, (b) 2.23 (1.94) aF, 130 (2960) $\mathrm{M} \Omega$, and $0.06 \mathrm{e}$, (c) 0.89 (1.16) $\mathrm{aF}, 70(950) \mathrm{M} \Omega$, and $-0.21 e$ accordingly. The fitting curves are displaced vertically for clarity. play the atomiclike electronic state, ${ }^{15}$ the energy levels of a small metal particle are quantized from the bulk energy-band structures. With comparable energy scales for the Coulomb charging energy and the internal quantum energy level spacing, the $I-V$ curve can exhibit a complicated behavior, as above. ${ }^{2}$ Although multiple junctions may also cause the irregular staircases, ${ }^{14}$ the possibility is low here. In the experiment, we only observed the staircases above the Au particles but Ohmic characteristics on SAM with no particles, which indicates that the tip did not pick up a $\mathrm{Au}$ particle to form a multiple junction above another being measured Au particle on SAM/Au.

In order to understand the quantum size effect qualitatively, we consider a simple model of a particle in a box for independent electrons. The mean spacing of the energy levels can be estimated from the volume $V$ of the Au particles by $\delta E=2 \pi^{2} \hbar^{2} /\left(m_{e} k_{F} \vee\right)$, where $m_{e}$ is the electron mass, and $k_{F}$ is the Fermi wave vector $\left(1.2 \times 10^{8} \mathrm{~cm}^{-1}\right.$ for $\mathrm{Au}) .^{11,37,38}$ For a gold particle $1.5 \mathrm{~nm}$ in diameter, the mean spacing of energy levels is expected to be $\delta E \sim 70 \mathrm{meV}$ by assuming the particle as a sphere. For a gold particle of 1 $\mathrm{nm}, \delta E \sim 240 \mathrm{meV}$. Our measured voltage spacing (except for the zero conductance gap) for the sample with a nominal gold core size of $1.8 \mathrm{~nm}$ falls into this range, as shown in Fig. 3(d). Thus, the very large zero conductance gap observed for the small Au clusters seems to result mainly from quantum size effect that opens up an energy gap near the Fermi level for the particles. The coated ligand molecules, especially the head group of $S$ atoms may further reduce the volume in which the free electrons can travel and give a larger energy gap for the gold particle than the above energy spacing estimated for pure Au particles. Further theoretical calculation is still undergoing.

From the above estimate, we see that the energy-level spacing depends on the volumes of the particles. In Fig. 4, in addition to the result from Fig. 3(d), we show another two $d I / d V$ curves with different widths of zero conductance and different voltage spacings between adjacent current steps, taken for particles on the sample with a nominal particle size of $1.8 \mathrm{~nm}$. The actual particle size for this sample ranges from 1 to $3 \mathrm{~nm}$. Thus, the observed different voltage spacing reflects the different quantized energy levels due to different particle sizes. Both on the positive-bias side and the negative-bias side of Fig. 4, we observed nearly similar characteristics as those for InAs quantum dots ${ }^{15}$ and $\mathrm{Au},{ }^{18}$ which displays the quantized energy levels. To further identify the quantized energy levels with the exact particle size, nanoparticles with a much narrower size distribution are required. We are currently making an effort to obtain these better controlled particles by fractionation. ${ }^{18,26}$

By adjusting the tip-particle distance, the capacitance $C_{2}$ can be tuned. In Fig. 5(a), a series of $I-V$ curves taken for a particle with a nominal diameter of $\sim 15.2 \mathrm{~nm}$ were shown. The tip-particle distance was controlled by the set point current from 2 to $25 \mathrm{nA}$ with a constant set point voltage of $+1.5 \mathrm{~V}$. The measured $I-V$ curves were reversible and highly repeatable, which indicates no damage occurs to the particle due to the relatively high tunneling current or the short tip-particle distance. In Fig. 5(a), instead of using $I$ as 


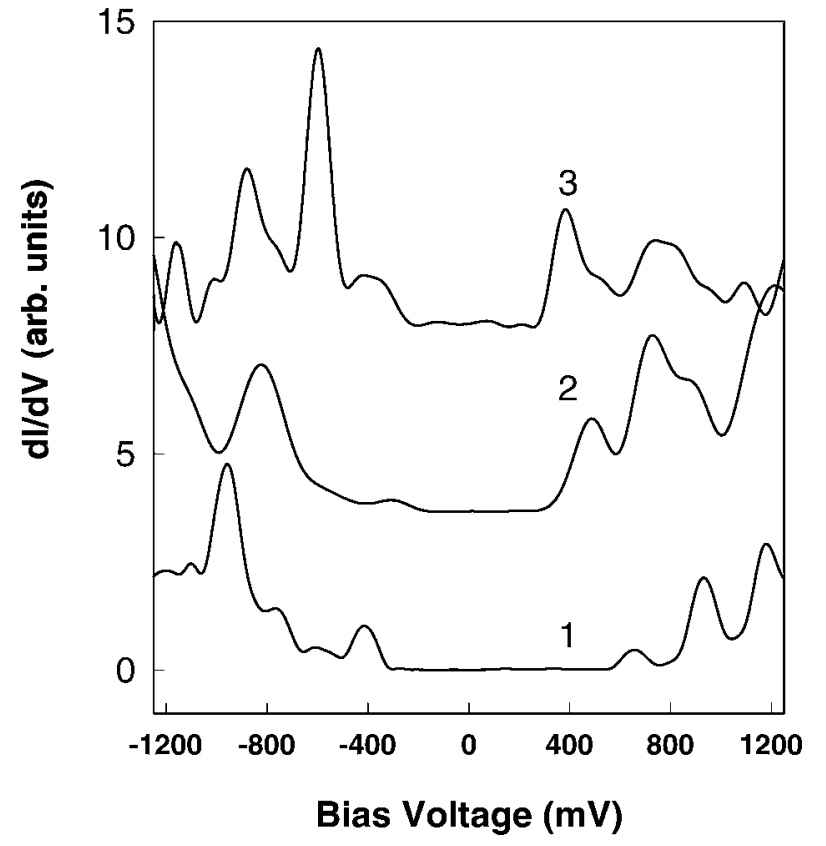

FIG. 4. Several digital differential tunneling conductances $d I / d V$ taken from gold particles on a sample with a nominal core size of $1.8 \mathrm{~nm}$ in diameter. Curves are displaced vertically for clarity.

the vertical axis, we use the product of the tunneling current $I$ and the asymptotic resistance $R$, where $R$ is estimated from the slope of the $I-V$ curve. To see the change of step spacing clearly, the digital differential conductances corresponding to the $I-V$ curves in Fig. 5(a) are plotted in Fig. 5(b). The average spacing of the peaks in Fig. 5(b) are measured as $42.7,37.4,36.3$, and $35.7 \mathrm{meV}$ as the set point current increases from 2 to $25 \mathrm{nA}$. This clearly shows that adjusting the tip-particle distance can tune the Coulomb charging energy. With the increase of set point current, the tip-particle distance decreases. As a result, $C_{2}$ becomes larger, but $R_{2}$ becomes smaller. In order to determine the junction parameters, we at first fitted the curve taken at a set point current of $2 \mathrm{nA}$ by the orthodox theory. ${ }^{3,4}$ The fitting parameters are $C_{1}=4.08 \mathrm{aF}, \quad C_{2}=3.95 \mathrm{aF}, \quad R_{1}=69 \mathrm{M} \Omega$, and $R_{2}$ $=1700 \mathrm{M} \Omega$, with a fractional residual charge of $Q_{0}$ $=0.15 \mathrm{e}$. As mentioned above, the fitting result of $C_{1}$ is very close to the calculated value of $3.78 \mathrm{aF}$. Assuming that the particle-electrode junction and hence, $C_{1}$ and $R_{1}$ remain unchanged, we can then fit $C_{2}, R_{2}$, and $Q_{0}$ for the rest of the $I-V$ curves at other set point currents. The fitting parameters are listed in Table I. At a set point current of $15 \mathrm{nA}$, the fitting value of $C_{2}$ reaches $4.57 \mathrm{aF}$, which is larger than the calculated value by assuming a tip-particle distance of $1 \mathrm{~nm}$ (correspond to $C_{2}=4.3 \mathrm{aF}$ ), implying that the STM tip starts to compress the ligand film. The fitting curves are also plotted in Fig. 5(a) in solid lines. They agree well with the experimental data, indicating that the change of voltage spacing in the staircases is mainly from the variation of the tipparticle distance. For small particles, the capacitance $C_{2}$ can also be tuned. Generally, the tuning range for small particles is relatively smaller than for large ones.

One may also notice from Table I that the fitted residual
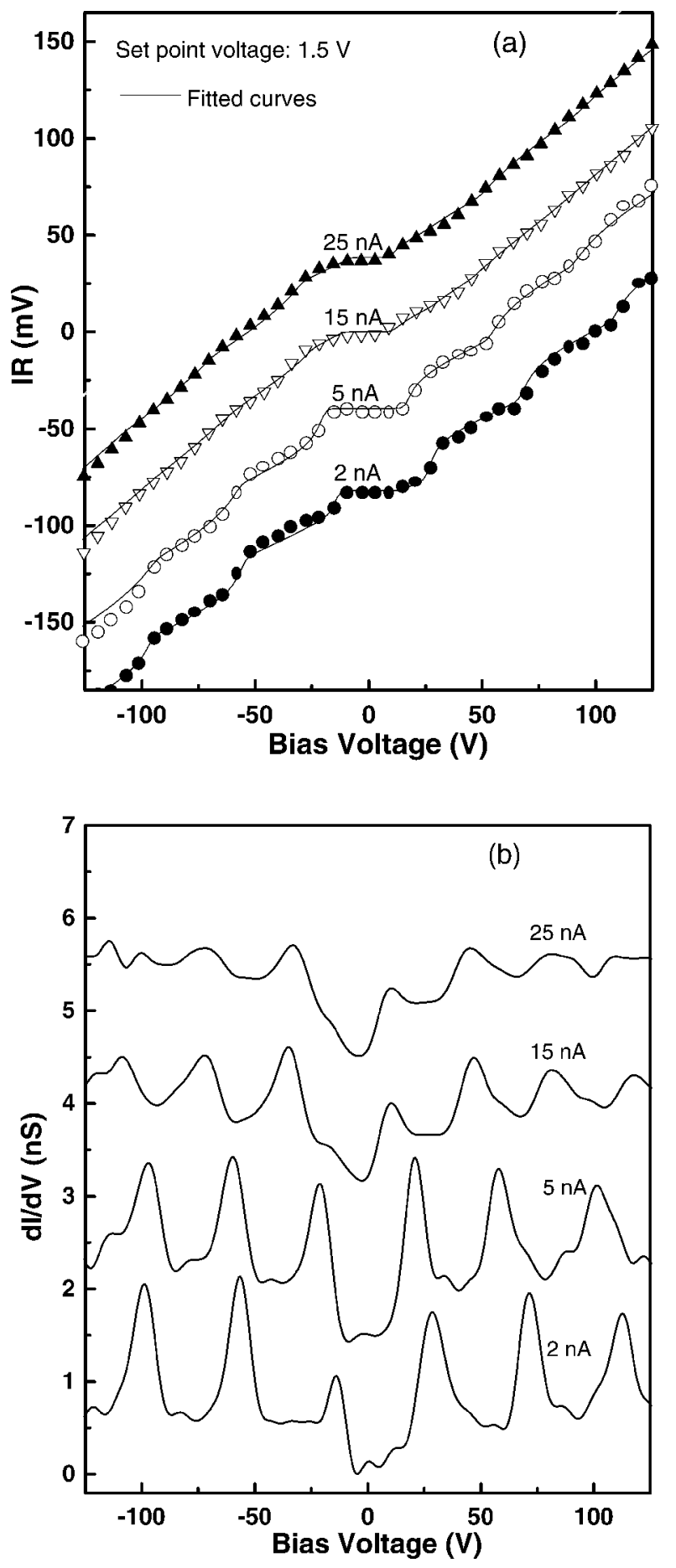

FIG. 5. $I-V$ curves (a) and their digital differential tunneling conductance (b) taken for a gold particle with a nominal size of $15.2 \mathrm{~nm}$ at different set point currents. Curves are displaced vertically for clarity.

charge $Q_{0}$ for the same particle changes from positive to negative and then back to positive as the tip-particle distance decreases. This is perhaps due to a fluctuation mechanism. ${ }^{13}$ As reported by Dubois et al. ${ }^{13}$ even for the same particle at a given tip-particle separation there still existed a fluctuation of residual charge in a relatively wide range in consecutive measurements. From the relationship given by Hanna and 
TABLE I. Fitting parameters $C_{2}, R_{2}$, and $Q_{0}$ at different set point current for a $\mathrm{Au}$ particle with nominal size of $15.2 \mathrm{~nm}$ by fixing $C_{1}=4.08 \mathrm{aF}, R_{1}=69 \mathrm{M} \Omega$.

\begin{tabular}{lcccc}
\hline \hline Set point current(nA) & 2 & 5 & 15 & 25 \\
\hline$C_{2}(\mathrm{aF})$ & 3.95 & 4.39 & 4.57 & 4.94 \\
$R_{2}(\mathrm{M} \Omega)$ & 1700 & 650 & 103 & 17.4 \\
$Q_{0}$ & $0.15 e$ & $-0.05 e$ & $-0.2 e$ & $0.2 e$ \\
\hline \hline
\end{tabular}

Tinkham $^{3}$ that the residual charge is proportional to the differences of the work functions between the electrodes, including the tip, the substrate, and the particle, it is not difficult to understand that the change of surface state under the external field may cause residual charge fluctuation. For example, the diffusion of atoms at the apex of the tip, which may not cause a large change in capacitance, may cause a change in the work function, and thus, an obvious change in the residual charge.

\section{CONCLUSION}

We have investigated the tunable SET behavior by STM/ STS at $4.2 \mathrm{~K}$. By using narrowly dispersed ligand-stabilized gold particles, the STM/STS results correlated well with the particle sizes, which were characterized by HREM. Only the $\mathrm{CB}$ effect, e.g., equidistant spaced staircases, was observed in the $I-V$ curves for gold particles with sizes $>4.6 \mathrm{~nm}$, for which the fitting capacitance values complied very well with the calculated results. For gold particles of $\sim 1.8 \mathrm{~nm}$, irregularly spaced current steps were observed in the $I-V$ curves, which indicated that both the $\mathrm{CB}$ effect and the quantum size effect made contributions. By adjusting the tip-particle junction, the capacitance of $C_{2}$ of the tip-particle junction was tuned from smaller than $C_{1}$ of the particle-electrode junction to larger than $C_{1}$, and consequently was the SET behavior finely tuned. The tuning behavior can be useful for developing tunable SET devices.

\section{ACKNOWLEDGMENTS}

We gratefully acknowledge the financial support from the National Science Foundation of China and the "QiuShi', Foundation of Hong Kong. X.X. also wishes to acknowledge the financial support from the Hong Kong University of Science and Technology through the William Mong Solid State Cluster Laboratory and the High Impact Area Fund.

${ }^{12}$ C.-S. Jiang, T. Nakayama, and M. Aono, Appl. Phys. Lett. 74, 1716 (1999).

${ }^{13}$ J. G. A. Dubois, J. W. Gerritsen, S. E. Shafranjuk, E. J. G. Boon, G. Schmid, and H. van Kempen, Europhys. Lett. 33, 279 (1996).

${ }^{14}$ E. Bar-Sadeh, Y. Goldstein, C. Zhang, H. Deng, B. Abeles, and O. Millo, Phys. Rev. B 50, 8961 (1994).

${ }^{15}$ U. Banin, Y. W. Cao, D. Katz, and O. Millo, Nature (London) (London) 400, 542 (1999).

${ }^{16}$ S.-T. Yau, P. Mulvaney, W. Xu, and G. M. Spinks, Phys. Rev. B 57, R15 124 (1998).

${ }^{17}$ L. F. Chi, M. Hartig, T. Drechsler, Th. Schwaack, C. Seidel, H. Fuchs, and G. Schmid, Appl. Phys. A: Mater. Sci. Process 66, S187 (1998).

${ }^{18}$ S. W. Chen, R. S. Ingram, M. J. Hostetler, J. J. Pietron, R. W. Murray, T. G. Schaaff, J. T. Khoury, M. M. Alvarez, and R. L. Whetten, Science 280, 2098 (1998).

${ }^{19}$ Jun-ichi Shirakashi and Kazuhiko Matsumoto, Appl. Phys. Lett. 72, 1893 (1998).

${ }^{20}$ K. Matsumoto, Physica B 227, 92 (1996).

${ }^{21}$ David L. Klein, Richard Roth, Andrew K. L. Lim, A. Paul Alivisatos, and Paul L. McEuen, Nature (London) (London) 389, 699 (1997).

${ }^{22}$ T. Sato and H. Ahmed, Appl. Phys. Lett. 70, 2759 (1997).

${ }^{23}$ K. Matsumoto, M. Ishii, K. Segawa, Y. Oka, B. J. Vartanian, and J. S. Harris, Appl. Phys. Lett. 68, 34 (1996).

${ }^{24}$ D. L. Klein, P. L. McEuen, J. E. B. Katari, R. Roth, and A. P. Alivisatos, Appl. Phys. Lett. 68, 2574 (1996).

${ }^{25}$ R. S. Ingram, M. J. Hostetler, R. W. Murray, T. G. Schaaff, J. T. Khoury, R. L. Whetten, T. P. Bigioni, D. K. Guthrie, and P. N. First, J. Am. Chem. Soc. 119, 9279 (1997).

${ }^{26}$ R. L. Whetten, J. T. Khoury, M. M. Alvarez, S. Murthy, I. Vezmar, Z. L. Wang, P. W. Stephens, C. L. Cleveland, W. D. 
Luedtke, and U. Landman, Adv. Mater. 8, 428 (1996).

${ }^{27}$ M. Brust, D. Bethell, D. J. Schiffrin, and C. J. Kiely, Adv. Mater. 7, 795 (1995).

${ }^{28}$ M. Brust, M. Walker, D. Bethell, D. J. Schiffrin, and R. Whyman, J. Chem. Soc. Chem. Commun. 801 (1994).

${ }^{29}$ M. J. Hostetler, J. E. Wingate, C.-J. Zhong, J. E. Harris, R. W. Vachet, M. R. Clark, J. D. Londono, S. J. Green, J. J. Stokes, G. D. Wignall, G. L. Glish, M. D. Porter, N. D. Evans, and R. W. Murray, Langmuir 14, 17 (1998).

${ }^{30}$ C. B. Murray, D. B. Norris, and M. G. Bawendi, J. Am. Chem. Soc. 115, 8706 (1993).

${ }^{31}$ A. P. Alivisatos, Science 271, 933 (1996).

${ }^{32}$ M. P. Pileni, Langmuir 13, 3266 (1997).
${ }^{33}$ N. Camillone III, T. Y. B. Leung, P. Schwartz, P. Eisenberger, and G. Scoles, Langmuir 12, 2737 (1996).

${ }^{34}$ Thin Films-Self-Assembled Monolayers of Thiols, edited by A. Ulman (Academic, San Diego, 1998), pp. 121-125.

${ }^{35}$ M. A. Rampi, O. J. A. Schueller, and G. M. Whitesides, Appl. Phys. Lett. 72, 1781 (1998).

${ }^{36}$ C. Schönenberger, J. Jorritsma, J. A. M. Sondag-Huethorst, and L. G. J. Fokkink, J. Phys. Chem. 99, 3259 (1995).

${ }^{37}$ D. V. Averin and A. N. Korotkov, J. Low Temp. Phys. 80, 173 (1990).

${ }^{38}$ Charles Kittel, Introduction to Solid State Physics, 7th Ed. (Wiley, New York, 1996), p. 150. 